\title{
Prevalencia de erosión dental en estudiantes de 12 a 16 años utilizando Basic Erosive Wear Examination (BEWE) en una institución educativa pública peruana.
}

\author{
Luis Angel Hayakawa Lastarria, ${ }^{1}$ \\ Angie Gallo Oropeza, ${ }^{1}$ \\ Leslie Casas-Apayco. ${ }^{2}$
}

\section{Resumen}

Introducción: El objetivo de este estudio fue determinar la prevalencia y la distribución de erosión dental en estudiantes de 12 a 16 años de edad de la institución educativa pública María Auxiliadora del distrito de Chorrillos, Lima-Perú. Materiales y métodos: Se evaluaron 382 estudiantes de una institución educativa pública peruana con el índice de diagnóstico de erosión dental Basic Erosive Wear Examination (BEWE). Después de la evaluación clínica, se obtuvieron los puntajes por sextantes, y posteriormente fueron sumados para obtener en un puntaje acumulativo total. Los datos fueron analizados mediante estadística descriptiva y para relacionar la erosión con las variables género y edad se aplicó la prueba de Chi cuadrado y Anova (una cola) $(p<0,05)$. Resultados: La prevalencia de erosión dental fue de $12.04 \%$. En cuanto a su distribución, la edad más representativa fue el grupo de 16 años (28.26\%), el género femenino fue el más frecuente $(54.3 \%)$, y en cuanto a la localización por maxilar, el inferior resultó predominante $(54.35 \%)$ en los dientes posteriores, (56.52\%) y caras oclusales (65\%). Conclusión: Del total de estudiantes evaluados, el 12.04\% presentó erosión dental. Asimismo, se encontró mayor distribución de erosión dental en el género femenino y en los estudiantes de 16 años de edad, sin embargo no hubo significancia estadística.

Palabras clave: Erosión dental, prevalencia, salud pública, jóvenes.

1. Alumno de la Carrera de Odontología, Facultad de Ciencias de la Salud, Universidad Peruana de Ciencias Aplicadas-UPC, Lima, Perú.

2.Profesor investigador y Docente del área de Odontología Restauradora en la facultad de Odontología de la Universidad Peruana de Ciencias Aplicadas-UPC, Lima, Perú. 


\section{Prevalência da erosão dental em estudantes de 12 a 16 anos de idade utilizando Basic Erosive Wear Examination (BEWE) numa escola pública peruana.}

\section{Resumo}

Introdução: $\mathrm{O}$ objetivo deste estudo foi determinar a prevalência e a distribuição da erosão dental em estudantes de 12 a 16 anos de idade de uma escola pública Maria Auxiliadora (Distrito Chorrillos), Lima-Perú. Materiais e métodos: 382 estudantes de uma escola pública peruana foram avaliados com o Basic Erosive Wear Examination (BEWE), índice de diagnóstico de erosão dental. Após da avaliação clínica, a pontuação foi obtido por sextantes, e posteriormente foram somados para obter a pontuação total. Os dados foram analisados mediante a análise estatística descritiva. Para erosão dentária, as variaveis gênero e idade aplicou-se o teste Chi quadrado e Anova a uma significancia de $\mathrm{p}>0,05$. Resultados: A prevalência da erosão dental foi de $12.04 \%$. De acordo à distribuição, a idade mais representativa foi o grupo de 16 anos (28.26\%), o gênero feminino o mais frequente $(54.3 \%)$, e pela localização, o maxilar inferior resultou predominante $(54.35 \%)$, nos dentes posteriores $(56.52 \%)$, e nas faces colusais. (65\%). Conclusão: Do total de estudante avaliados, o $12.04 \%$ apresentou erosão dental. Também, encontrou-se maior distribuição de erosão dental no gênero feminino e nos estudantes de 16 anos de idade, porém não houve diferença estatisticamente significante.

Palavras-chaves: Erosão dental, prevalência, saúde pública, estudantes.

Original article

\section{Dental erosion in 12 to 16 years old school students using Basic Erosive Wear Examination (BEWE) index}

\begin{abstract}
Introduction: The aim of this study was to determine the prevalence and distribution of dental erosion in 12 to 16 year old students from "María Auxiliadora" public school at Chorrillos, Lima-Perú. Materials and methods: A total of 382 students were clinically exanimated for dental erosion
\end{abstract}

using the Basic Erosive Wear Examination (BEWE). After the clinical examination, scores were obtained by sextants. They were then summed to produce in a accumulative score. The data was analyzed by descriptive statistics to relate the erosion with the sex and age. The Chi squared and Anova test (oneway) were used $(p<0,05)$. Results: The prevalence of dental erosion 
was $12.04 \%$ and as to its distribution, the most representative age was the group of 16 years $(28.26 \%)$, female were most frequent $(54.3 \%)$, the predominant location was the lower maxilary (54.35\%) posterior teeth (56.52\%) oclusal surfaces (65\%). Conclusions: Of the total number

\section{Introducción}

La erosión dental es la pérdida de tejido dentario de manera irreversible por una exposición constante a medios ácidos no bacteriogénicos que puede resultar en problemas funcionales, estéticos y psicológicos. ${ }^{1-6}$ Actualmente, a nivel mundial, se han realizado múltiples estudios que determinan una prevalencia considerable de erosión dental en poblaciones de diferentes etnias y rangos de edad, lo que hace que dicha enfermedad sea un problema de salud actual..$^{7-26}$

La etiología de la erosión dental es multifactorial, sin embargo pueden identificarse dos tipos de factores: los intrínsecos y extrínsecos. Los intrínsecos son los generados por nuestro propio organismo, éstos pueden ser desórdenes del tracto digestivo como reflujo gastroesofágico y/o trastornos alimenticios (bulimia, anorexia). Los factores extrínsecos están asociados a la ingesta de bebidas y/o alimentos ácidos como frutas cítricas incluidas en la dieta, bebidas con $\mathrm{pH}$ ácido e incluso medicamentos. ${ }^{2-6}$

La característica clínica inicial de la erosión dental es la pérdida del brillo del esmalte. Posteriormente, se genera of students evaluated, $12.04 \%$ had dental erosion. A greater distribution of dental erosion in the female and in students of 16 years of age was found, but there was no statistical significance.

Key words: Dental erosion, prevalence, public health, young population.

una mayor translucidez de la dentina $y$ cuando esta se encuentra comprometida, presenta sensibilidad o dolor a los cambios térmicos. ${ }^{2-5}$ Por otro lado, la manifestación clínica difiere según el tipo de diente (anterior o posterior), así como la cara comprometida (oclusal o cara libre). ${ }^{3,5}$ En los dientes posteriores con presencia de erosión dental se pueden encontrar lesiones socavadas en la cara oclusal con un color más amarillento por la exposición de dentina, a diferencia de los dientes anteriores donde se encontrará un superficie lisa, aplanada y brillante, con pérdida del cíngulo.,

En la actualidad, gran parte de los odontólogos desconocen cómo diagnosticar y proceder frente a la presencia de esta enfermedad en sus estadios más tempranos. Por tal motivo, se han desarrollado diversos índices de diagnóstico de erosión dental, los cuales clasifican las lesiones erosivas de acuerdo a criterios como presencia, severidad y localización, etc. ${ }^{7-32}$ Uno de ellos, es el índice Basic Erosive Wear Examination (BEWE), determinado por Bartlett del Reino Unido, Ganss de Alemania y Lussi de Suiza en 2008. Este índice es de uso fácil y sencillo, además ubica el diagnóstico de erosión dental en rangos simplificados que permiten determinar un nivel de riesgo 
de acuerdo a la severidad diagnosticada, relacionándola con un plan de tratamiento preventivo y/o restaurador. ${ }^{32}$

Estudios epidemiológicos realizados en diferentes países reportan distintas prevalencias de erosión dental. ${ }^{7-25}$ (Tabla 1) Sin embargo, en el Perú existen pocos estudios de prevalencia de erosión dental en población infantil. En el 2016, Baltuano et al. realizaron un primer estudio de prevalencia de erosión dental utilizando el índice BEWE en el rango de 6 a 12 años, encontrando una prevalencia de $9.31 \%$. Partiendo de este dato es que se propone determinar la prevalencia de erosión dental en un rango de 12 a 16 años y verificar el aumento o la disminución de la presencia de la lesión utilizando el mismo índice.

Por tal motivo, el objetivo de este estudio es determinar la prevalencia de erosión dental en el rango de edad de 12 a 16 años en una institución educativa pública de Perú.

\section{Materiales y Métodos}

Este estudio fue de tipo descriptivo y transversal. Se realizó en una institución pública educativa del distrito de Chorrillos ubicada al sur de la ciudad de Lima-Perú durante los meses de marzo a julio del 2017. La institución fue seleccionada por encontrarse cerca a la ubicación geográfica del centro universitario, y ser una institución educativa pública y representativa de la región. El distrito se encuentra habitado en su mayoría por familias de nivel socioeconómico medio-bajo. El colegio contaba con servicios básicos de agua, luz y desagüe.

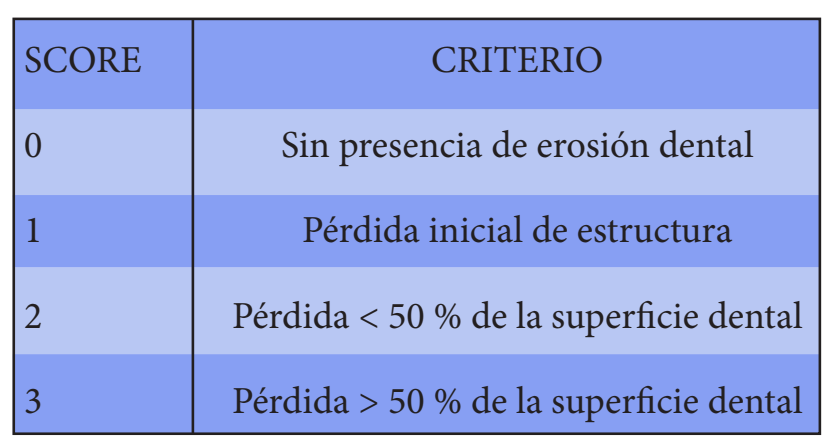

Tabla 1. Índice Basic Erosive Wear Examination (BEWE).

La población total del nivel secundario (12-16 años) estaba conformada por 440 estudiantes. Inicialmente se determinó un tamaño de muestra, usando la fórmula de estimación de una proporción, considerando un nivel de confianza de $95 \%$, precisión de $5 \%$ y una proporción esperada de $13 \%$ obtenida de un artículo previo de Peres et al., ${ }^{11}$ resultando en 174 estudiantes. Esto se realizó con el objetivo de establecer un número mínimo de alumnos que debían ser evaluados para que el estudio tuviera relevancia significativa. Posteriormente, se realizó un censo para abarcar la totalidad de la población de 440 , sin embargo, debido a los criterios de inclusión y exclusión 58 estudiantes no participaron; 40 por no presentar el asentimiento o consentimiento, 16 por no estar dentro del rango de edad evaluado y 2 por presentar aparatología de ortodoncia, quedando un total de 382 estudiantes.

Los criterios de inclusión fueron estudiantes entre 12 a 16 años de edad, que firmaran el asentimiento informado y cuyos padres firmaran el consentimiento informado. Por otro lado, los criterios de exclusión fueron aquellos estudiantes que no desearan participar del estudio, portadores de aparatología ortodóntica, 
y por último aquellos que presentaron dientes afectados por defectos del desarrollo del esmalte (DDE) en sus manifestaciones más severas.

Este estudio fue aprobado por el comité de ética de la Universidad Peruana de Ciencias Aplicadas CEI/235-11-16.

Para la aplicación del índice BEWE, se realizó una calibración inter-examinador entre los dos investigadores que realizaron este estudio con un Kappa de Cohen (0.80). Así mismo, una calibración intra-examinador con el especialista en odontología restauradora y estética obteniéndose un Kappa de Cohen similar.

La evaluación clínica y diagnóstico BEWE se realizó en un área específica provista por la institución educativa. Se procedió a colocar una colchoneta sobre la mesa, cuya superficie se cubrió con papel craft al igual que la almohadilla, donde se realizó la evaluación clínica en posición supina. Los estudiantes realizaron un enjuagatorio bucal previo al examen clínico. Se utilizaron todas las medidas de bioseguridad (uniforme, mascarilla, guantes, lentes de protección, gorro) tanto por el operador como para el estudiante. Los dientes fueron evaluados mediante el uso de un equipo de examen clínico con la ayuda de un frontoluz, las superficies dentales fueron secadas con torundas de algodón y se registraron los puntajes encontrados de cada diente.

El examen clínico consistió en revisar las superficies vestibular, palatino, lingual y oclusal de cada diente registrando el valor BEWE por sextantes. Luego se colocó el puntaje más alto de cada sextante (puntaje 0 , puntaje 1 , puntaje 2, puntaje 3) según lo hallado. (Tabla 2). Posteriormente, los valores representativos de cada sextante fueron sumados para obtener un puntaje acumulativo total. (Tabla 3). Este puntaje acumulativo es correlacionado a la tabla de nivel de riesgo y tipo de tratamiento sugerido por el mismo índice BEWE2 (Figura 1). Las lesiones erosivas de algunos pacientes fueron registradas en fotografías digitales (cámara Lumix GF6 Lente 14-42) seleccionándose las más representativas para cada puntaje (Figura 2).

Al finalizar la evaluación clínica, se proporcionó a los estudiantes de la muestra, dípticos relacionados con la erosión dental, definición, signos, síntomas y las recomendaciones adecuadas para estos casos.

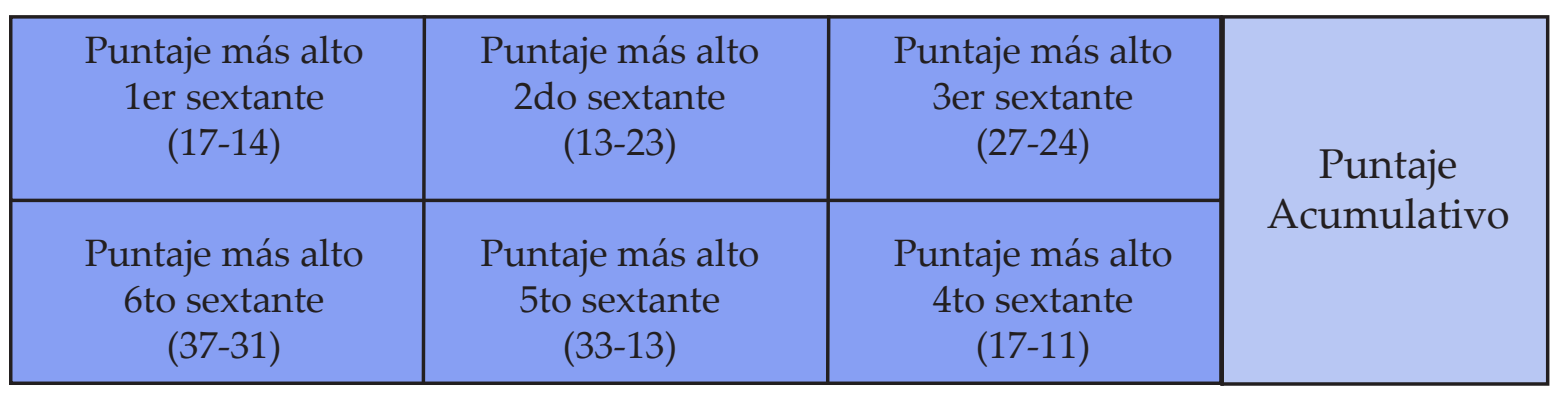

Tabla 2. Cálculo de Puntaje Acumulativo (adaptado de Bartlett et al. 2008). 


\begin{tabular}{|c|c|c|}
\hline $\begin{array}{l}\text { NIVEL DE } \\
\text { RIESGO }\end{array}$ & $\begin{array}{c}\text { PUNTAJE } \\
\text { ACUMULATIVO }\end{array}$ & MANEJO \\
\hline Ninguno & $\leq 2$ & $\begin{array}{l}\text { - Mantenimiento de rutina y observación } \\
\text { - Repetir en intervalos de } 3 \text { años }\end{array}$ \\
\hline Bajo & Entre 3 - 8 & $\begin{array}{l}\text { - Asistencia nutricional y de higiene oral, } \\
\text { mantenimiento y observación } \\
\text { - Repetir en intervalos de } 2 \text { años }\end{array}$ \\
\hline Moderado & Entre 9 - 12 & $\begin{array}{l}\text { - Higiene oral, orientación y evaluación nutricional, } \\
\text { identificar el/los factor(es) etiológico principal(es) } \\
\text { y desarrollar estrategias para eliminar el impacto. } \\
\text { - Considerar métodos de fluoración. } \\
\text { - Idealmente evitar la colocación de restauraciones } \\
\text { y monitorear el desgaste. } \\
\text { - Repetir en intervalos de } 6 \text { a } 12 \text { meses. }\end{array}$ \\
\hline Alto & $14 \mathrm{o}+$ & $\begin{array}{l}\text { - Higiene oral, orientación y evaluación nutricional, } \\
\text { identificar el/los factor(es) etiológico principal(es) } \\
\text { y desarrollar estrategias para eliminar tales impactos. } \\
\text { - Considerar métodos de fluoración. } \\
\text { - Idealmente evitar colocación de restauraciones } \\
\text { y monitorear el desgaste. } \\
\text { - Especialmente en casos de severa progresión, } \\
\text { considerar cuidado especial que pueda involucrar } \\
\text { restauraciones. } \\
\text { - Repetir en intervalos de } 6 \text { - } 12 \text { meses. }\end{array}$ \\
\hline
\end{tabular}

Tabla 3. Niveles de riesgo como guía para el manejo clínico (adaptado de Bartlett et al. 2008).

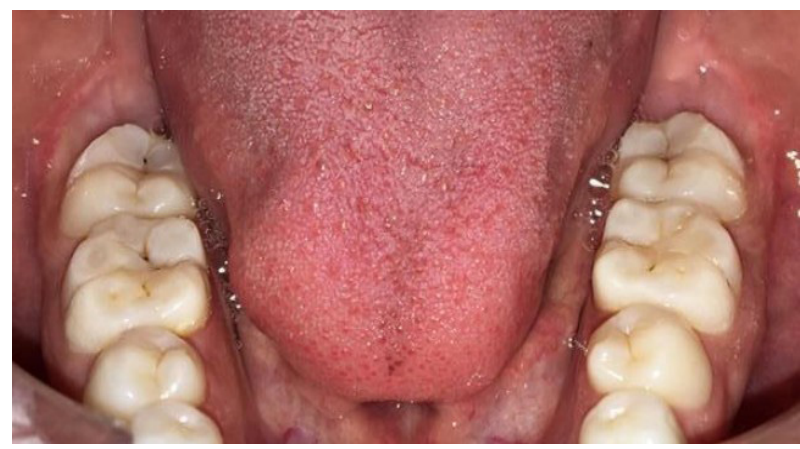

Figura 1. Erosión dental en 36 y 46 con hoyuelos y pérdida de brillo hoyuelos y pérdida de brillo (BEWE puntaje 2).

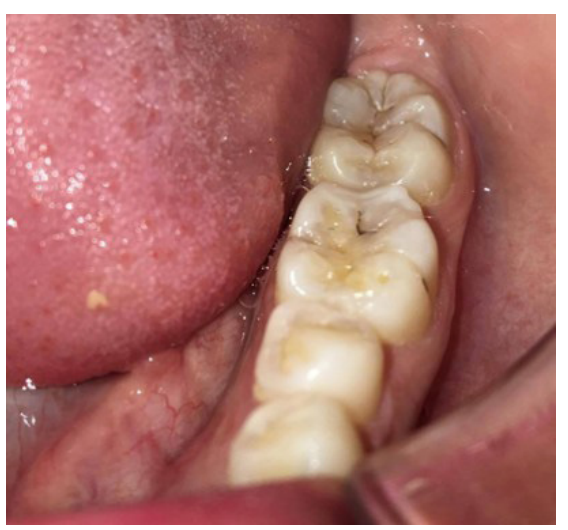

Figura 2. Erosión dental en pza Pieza 36 con mayor Translucidez del esmalte y hoyuelos (BEWE puntaje 2). 
Los datos fueron analizados mediante estadística descriptiva, frecuencia absoluta y relativa de la variable erosión dental según género, edad, tipo de diente, superficie y arco dental. Para el análisis bivariado se procedió a utilizar la prueba de Chi cuadrado para relacionar erosión dental con género y ANOVA a un criterio para determinar la asociación de la erosión dental con edad $(\mathrm{p}<0.05)$. (Microsoft Excel/ Stata ${ }^{\circledR}$ versión 13.0.)

\section{Resultados}

Se evaluaron 382 niños, de los cuales el $12.04 \%$ (46) presentaron signos de erosión dental y $87.96 \%$ (336) no presentaron esta enfermedad. La prevalencia y distribución de erosión dental según género, edad y arco dental en estudiantes de 12 a 16 años se muestra en la Tabla 4.

Del total de estudiantes evaluados, la prevalencia de erosión dental por puntajes en relación al género y la media y desviación estándar por edad se presenta en la Tabla 5. En cuanto a los valores obtenidos por puntajes, no hubo diferencias significativas en relación a la edad $(p=0,188)$ y género $(p=0,737)$.

Según el tipo de diente, la distribución de erosión dental en el arco superior fue mayor en la región posterior $(60 \%)$, al igual que en la mandíbula (64\%). Por otro lado, la superficie oclusal fue la más presente en el arco inferior (80\%) y en el arco superior (53\%). (Figura 3)

Tabla 4. Prevalencia y distribución de erosión dental según género, edad y arco dental en estudiantes de 12 a 16 años de la Institución Pública María Auxiliadora.

\begin{tabular}{|lccc|}
\hline \multicolumn{3}{c}{ Erosión } & \\
\hline Variable & Si & No & Total \\
Erosión dental & $46(12.04 \%)$ & $336(87.96 \%)$ & $382(100 \%)$ \\
\hline Género & & & \\
Femenino & $25(54.3 \%)$ & $162(48.21 \%)$ & $187(48.95 \%)$ \\
Masculino & $21(45.7 \%)$ & $174(51.79 \%)$ & $195(51.05 \%)$ \\
Total & $46(100 \%)$ & $336(100 \%)$ & $382(100 \%)$ \\
\hline Edad & & & \\
12 años & $10(21.74 \%)$ & $73(21.73 \%)$ & $73(21.73 \%)$ \\
13 años & $11(23.91 \%)$ & $68(20.24 \%)$ & $78(20.68 \%)$ \\
14 años & $6(13.04 \%)$ & $60(17.86 \%)$ & $66(17.28 \%)$ \\
15 años & $6(13.04 \%)$ & $63(18.75 \%)$ & $76(19.90 \%)$ \\
16 años & $13(28.26 \%)$ & $336(100 \%)$ & $382(100 \%)$ \\
Total & $46(100 \%)$ & $336(100 \%)$ & $336(87.96 \%)$ \\
\hline Arco dental & & 0 & $15(3.93 \%)$ \\
Ninguno & $0(0 \%)$ & 0 & $25(6.54 \%)$ \\
Superior & $15(32.61 \%)$ & 0 & $382(1.57 \%)$ \\
Inferior & $25(54.35 \%)$ & $336(100 \%)$ & \\
Ambos & $6(13.04 \%)$ & & \\
Total & $46(100 \%)$ & & \\
\hline
\end{tabular}


Tabla 5. Prevalencia y distribución de erosión dental según género, edad y arco dental en estudiantes de 12 a 16 años de la Institución Pública María Auxiliadora.

\begin{tabular}{|lcccccc|}
\hline \multicolumn{7}{c}{ Puntaje de erosión dental } \\
\hline Género & Puntaje 0 & Puntaje 1 & Puntaje 2 & Puntaje 3 & Total & p-Valor \\
Masculino & $174(89.23 \%)$ & $15(7.69 \%)$ & $6(3.08 \%)$ & 0 & $195(100 \%)$ & \\
Femenino & $162(86.63 \%)$ & $18(9.63 \%)$ & $7(3.74 \%)$ & 0 & $187(100 \%)$ & $0.737^{*}$ \\
Total & $336(87.96 \%)$ & $33(8.64 \%)$ & $13(3.4 \%)$ & 0 & $282(100 \%)$ & \\
\hline Edad & & & & & & \\
Total & $13.91 \pm 1.41$ & $13.78 \pm 1.5$ & $14.61 \pm 1.41$ & $0 \pm 0$ & $13.93 \pm 1.42$ & $0.188^{* *}$ \\
\hline
\end{tabular}

Test de Chi ${ }^{2 *}$ Anova one way** $(\mathrm{p}<0.005)$

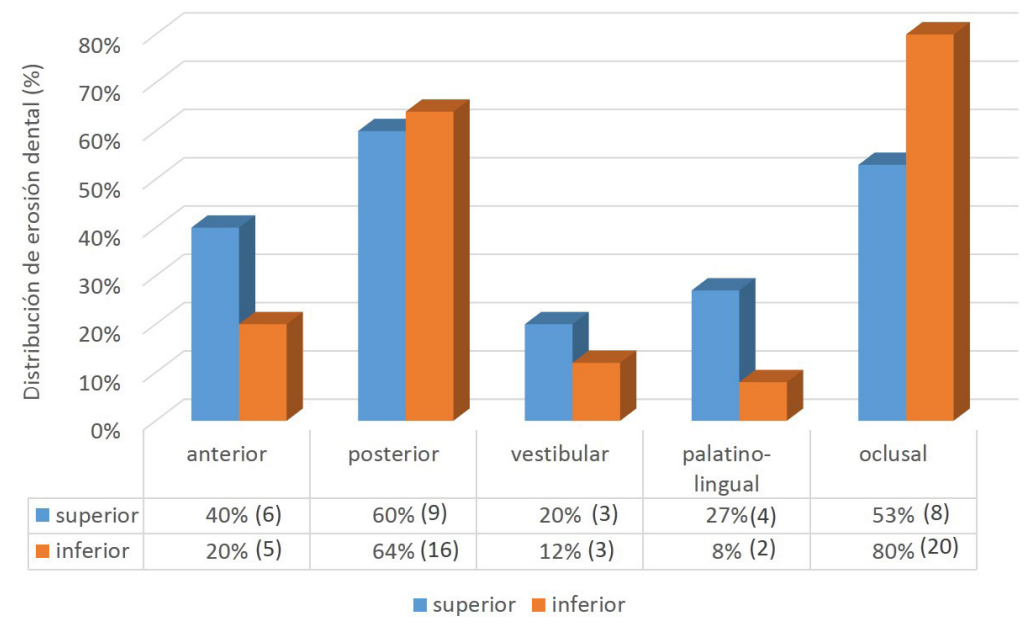

Figura 3. Distribución de erosión dental en tipos de dientes y superficies dentales según la ubicación en el arco dental.

Con respecto a la arcada dental y el puntaje encontrado, el puntaje 1 fue el más frecuente en el arco inferior (55\%) seguido del puntaje 2 (54\%). Asimismo, el puntaje 1 mostró una mayor distribución en la zona posterior (64\%). (Figura 4)

Al análisis de las fotografías clínicas registradas, se encontró que los primeros molares inferiores permanentes presentaron el mayor valor por sextante. Se pudo observar superficies oclusales socavadas con formas de hoyuelos y pérdida de brillo y en una de ellas se muestra la dentina por translucidez del esmalte. En dichas lesiones se otorgó un puntaje 2. 


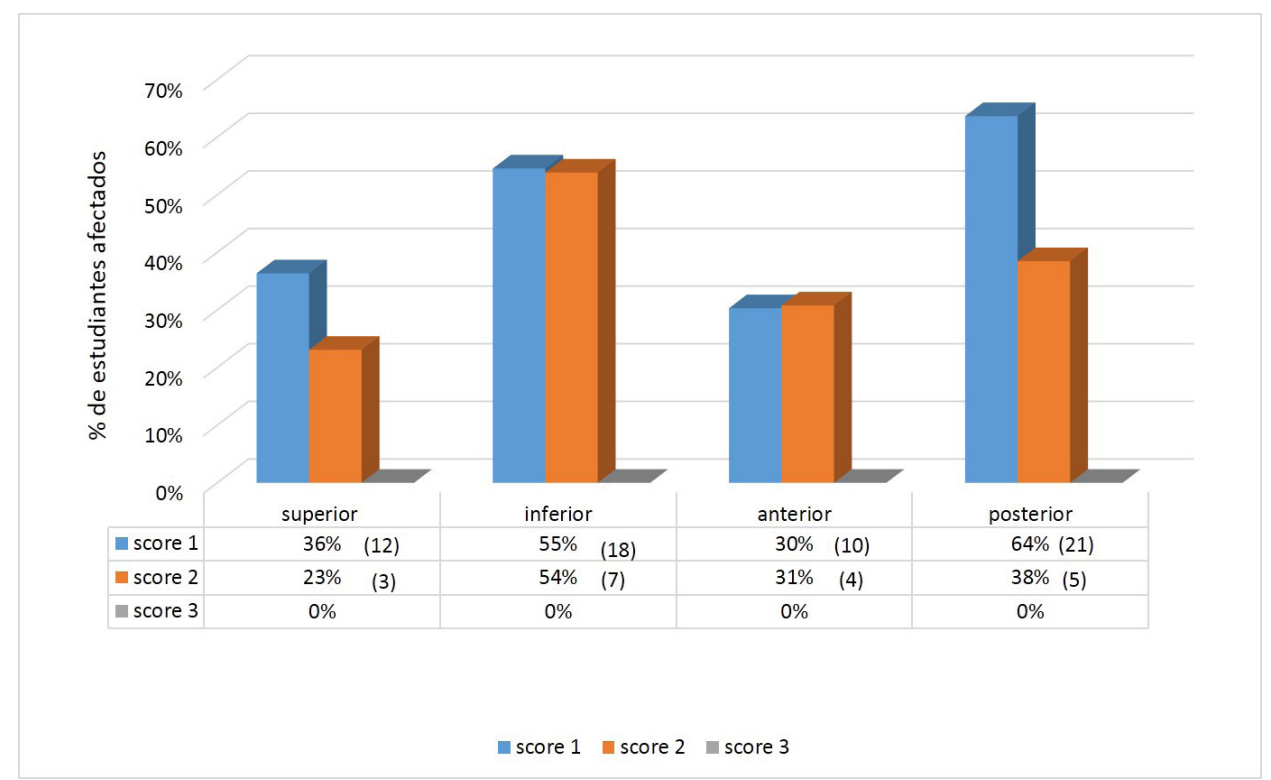

Figura 4. Distribución de erosión dental en tipos de dientes $y$ arcos dentales según la severidad de erosión por BEWE.

\section{Discusión}

En la actualidad existen pocos estudios en Latinoamérica sobre la prevalencia de erosión dental usando como método de diagnóstico el índice de BEWE, y aún menos los estudios realizados en el rango de edad de 12 a 16 años.

La prevalencia de erosión dental obtenida en el presente estudio es similar al 13\% reportado por Peres et al. ${ }^{11}$ en niños de 12 años en Brasil y al $8.9 \%$ de Kumar et al..$^{23}$ para edades comprendidas entre 11 y 14 años, en la ciudad de Udupi-India. ${ }^{11,23}$ No obstante, existen otras investigaciones como la de Loureiro et al, quienes encontraron $52.9 \%$ de prevalencia en niños de 12 años en Uruguay, y Zhang et $a l .{ }^{9}$ que registraron una mayor prevalencia de erosión dental en $75 \%$ de individuos de la misma edad en China. ${ }^{9,16}$ Esto puede ser explicado en primer lugar porque existen diferencias con respecto a los rangos de edades considerados ya que en ambos estudios de Loureiro et al. y Zhang et al. ${ }^{9}$ se limitaron a examinar un grupo de edad en particular como es el caso específico de 12 años de edad, a diferencia del presente estudio donde se incluyeron individuos de 12-16 años. En segundo lugar, si bien el escenario de exploración es una dentición permanente, el tiempo del diente en la cavidad oral indica mayor probabilidad de que el diente haya podido sufrir desgastes o alteraciones por erosión. Sin embargo, esto no quiere decir que a más edad exista mayor prevalencia de erosión, ya que existen estudios como el de Mantonanaki et al. realizado en Grecia, que mostró una prevalencia de $78.8 \%$ en niños de 5 años, así como Dugmore et al. quienes encontraron $59.7 \%$ de prevalencia en niños de 12 años en Inglaterra, $\mathrm{y}$ Tschammler et al. en Alemania encontraron una prevalencia de $31,3 \%$ en niños de 3 a 6 años de edad. ${ }^{10,13,22}$ En tercer lugar, se sabe que la erosión dental es una enfermedad multifactorial y los factores 
de riesgo cambian de acuerdo a los estilos de vida, nivel socioeconómico, hábitos alimentarios y costumbres, lo cual tendría relevancia al momento de comparar estos estudios antes mencionados, ya que son poblaciones con ubicaciones geográficamente distintas., ${ }^{3,10,33-35}$ Por último, se debe tener en cuenta que los estudiantes en instituciones privadas suelen presentar una cantidad superior en cuanto a la prevalencia de erosión con respecto a los que provienen de instituciones públicas, como sucedió en el estudio de Peres et al. ${ }^{11}$ Se puede inferir que el poder adquisitivo de los individuos que provienen de escuelas privada es mayor con respecto a los que provienen de alguna institución educativa pública. El poder adquisitivo alto permitiría a su vez la compra de alimentos y bebidas más elaboradas e industrializadas con $\mathrm{pH}$ ácido y potencialmente erosivo, que muchas veces son consideradas más agradables fomentando el consumo frecuente.

Por otro lado, la erosión dental fue mayor en las niñas que en los niños, resultado similar al del estudio de Wang et al. en niños de 12 y 13 años de edad en China. ${ }^{15}$, pero diferente de otros estudios donde la mayor distribución se dio en el género masculino. ${ }^{7,12,16}$ Sin embargo, sin que exista una explicación para estos hallazgos según género que no fueron estadísticamente significativos.

La distribución de la enfermedad fue mayor a los 16 años de edad, sin embargo no existe evidencia científica clara que explique que la erosión dental se presente con mayor frecuencia a una edad específica. Por ejemplo, Zhang et al 9 registraron una prevalencia de $75 \%$ en niños de 12 años, mientras que Dugmore et al, y Peres et al encontraron 59.7\% y $13 \%$ en la misma edad respectivamente, de igual manera sucede en diversos grupos de edades en otros estudios que muestran prevalencias diferentes entre las mismas edades..$^{9,11,13}$ Esto es probable debido a que los factores asociados a la enfermedad como el consumo de bebidas carbonatadas, alimentación propia de la región y/o presencia de desórdenes alimentarios y otras enfermedades gastroesofágicas, etc. pueden variar según la ubicación geográfica de los individuos y su ambiente.

En cuanto a la localización de la lesión en la cavidad oral, el arco inferior fue el lugar de mayor presencia de erosión dental, siendo la ubicación más específica las caras oclusales de dientes posteriores. Dichos hallazgos son semejantes a los encontrados por Baltuano et al, Wang et al, Arnadottir et al, Mulic et al, y Provatenou et al.,75,18,24,26 Por otro lado, otros investigadores encontraron una mayor presencia de la lesión en otras ubicaciones como por ejemplo Rusyan et al, encontraron mayor presencia de la enfermedad en los dientes anteriores de la misma forma que Mafla et al. ${ }^{17,25} \mathrm{La}$ ubicación de estas lesiones nos da un indicio de cómo se produjeron. El presente estudio indica que los molares mandibulares (cara oclusal) fueron los más afectados, lo cual evidencia un patrón de desgaste erosivo de carácter extrínseco en esta población, el cual se podría haber producido por una ingesta de bebidas carbonatadas y alimentos cítricos de un pH bajo. ${ }^{3,36}$

El puntaje más distribuido sin incluir el 0 , el cual significa la ausencia de la enfermedad, fue el puntaje 1 con $64 \%$ seguido del 2 con $38 \%$ en dientes posteriores, resultados similares al 
estudio de Rusyan et al, Muller-Bolla et $a l$, en esta investigación no se encontró la presencia de algún puntaje 3 resultado similar al de Zhang. ${ }^{8,9,17}$ Es comprensible que los dientes posteriores mandibulares (primeros molares permanentes) se encuentren más comprometidos debido a que la presencia de estos en la cavidad oral es más temprana que los demás dientes permanentes en su mayoría de casos, lo que indica que puede ser expuesto a este tipo de agresión por más tiempo.

La erosión dental es una enfermedad multifactorial, por lo tanto los factores de riesgo involucrados deben ser estudiados en cada población o región, tanto como la evaluación de los niveles de riesgo según BEWE para definir alternativas de tratamiento preventivo y/o restauradores, evitando así la progresión de la lesión.

\section{Conclusiones}

La prevalencia de erosión dental fue baja en el total de estudiantes evaluados $(12.04 \%)$ predominando en las niñas de 16 años, no siendo estadísticamente significativa.

Es importante realizar estudios epidemiológicos para determinar los factores asociados de esta enfermedad en la población peruana $\mathrm{u}$ otras poblaciones y de este modo poder establecer políticas de intervención que incluyan las etapas de diagnóstico, monitoreo, prevención y tratamientos restauradores de las lesiones erosivas.

\section{Referencias bibliográficas}

1. Smith WA, Marchan S, Rafeek RN. The prevalence and severity of non-carious cervical lesions in a group of patients attending a university hospital in Trinidad. J Oral Rehabil. 2008; 35: 128-34.

2. Álvarez C, Grille C. Revisión de la literatura: lesiones cervicales no cariogénicas. Cient Dent. 2008; 5:21524.

3. Infeld T. Dental erosion. Definition, classification and links. Eur J Oral Sci. 1996; 104: 151-5.

4. Almeida J, Araujo E, Baratiere L, Widmer N. Dental Erosion: understanding this pervasive condition. J Esthe Dent. 2011; 23: 205-16.

5. Picchi L, Aranda P, Martines B, Magalhaes A. Dental erosion: an overview on definition, prevalence, diagnosis and therapy. Braz Dent Sci.2013; 16: 06-17.

6. Milosevic A. Eating disorders and the dentist. Br Dent J. 1999; 186: 109-13.

7. Baltuano K, Flores K, Farfán M, Casas L. Prevalencia de erosión dental en niños de 6 a 12 años utilizando el índice Basic Erosive Wear Examination (BEWE). Rev Latinoam Odontoped. 2016; 6:17-22.

8. Muller-Bolla M, Courson F, Smail-Faugeron V, Bernardin T, Lupi-Péqurier L. Dental erosion in French adolescents. BMC Oral Health. 2015; 15: 147.

9. Zhang S, Chau AM, Lo EC, Chu CH. Dental caries and erosion status of 12 years old Hong Kong children. BMC Public Health. 2014 8; 14:7.

10. Mantonanaki M, Koletsi H, Mamai E. Papaioannou W. Dental erosion prevalence and associated risk indicators among preschool children in Athens, Greece. Clin Oral Invest. 2013; 17(2): 585-93.

11. Peres KG et al. Dental erosion in 12- year-old schoolchildren: a cross-sectional study in southern Brazil. Int J Paediatr Dent.2005; 15: 249-55.

12. Fajardo M, Mafla A. Diagnóstico y epidemiología de erosión dental. Salud UIS. 2011; 43: 179-89.

13. Dugmore CR, Rock WP. The progression of tooth erosion in a cohort of adolescents of mixed ethnicity. Int J of Paediatr Dent 2003; 13:295-303.

14. Deery C, Wagner ML, Longbotton C, Simon A, Nugent ZJ. The prevalence of dental erosion in a United 
States and a United Kingdom sample of adolescents. Pediatr Dent 2000; 22: 505-510.

15. Wang P, Lin HC, Chen JH Liang HY. The prevalence of dental erosion and associated risk factors in 12-13-year-old school children in Southern China. BMC public Health. 2010; 12: 478.

16. Loureiro L, Fabruccini A, Alves L, Alvarez R, Maltz M. Erosive Tooth Wear among 12-year old Schoolchildren: A population-based cross-sectional study in Montevideo, Uruguay. Caries Res. 2015; 49: 216-25.

17. Rusyan et al. Epidemiological study of prevalence and risk factors for dental erosions among polish young adults. Pol Merkur Lekarski. 2016; 40: 308-13.

18. Arnadottir IB, Holbrook WP, Eggertsson H, Gudmudsdottir H, Jonsson SH, Gudlaugsson JO, Saemundsson Sr, Eliasson ST, Agustsdottir H. Prevalence of dental erosion in children: a national survey. Community Dent Oral Epidemiol 2010; 38:521-6.

19. Salas MMS, Vargas-Ferreira F, Ardenghi TM, Peres KG, Huysmans MD, Demarco FF. Prevalence and Associated factors of Tooth Erosion in 8- to 12-year-old Brazilian Schoolchildren. J Clin Pediatr Dent. 2017; 41: 343-50.

20. Chu CH, Ng A, Chau AM, Lo EC. Dental Erosion and Caries Status of Chinese University Students. Oral Health Prev Dent. 2015; 13: 237-44.

21. Aidi H, Bronkhorst E, Truin G. A longitudinal study of tooth erosion in adolescents. J Dent Res. 2008; 87: 731-5.

22. Tschammler C, Müller-Pflanz C, Attin T, Müller J, Wiegand A. Prevalence and risk factors of erosive tooth wear in 3-6 year old German kindergarten children-A comparison between 2004/05 and 2014/15. J Dent. 2016; 52: 45-49.

23. Kumar S, Acharya S, Mishra P, Debnath N, Vasthare R. Prevalence and risk factors for dental erosion among 11-to 14-years old school children in South India. J Oral Sci. 2013; 55: 329-36.

24. Mulic A, et al. Dental erosion: Prevalence and severity among 16-year-old adolescents in Troms, Norway. Eur J Paediatr Dent. 2016; 17: 197-201.

25. Mafla A, et al. Prevalence and extrinsic risk factors for dental erosion in Adolescents. J Clin Pediatr Dent. 2017; 41(2).

26. Provatenou E, Kaklamanos EG, Kevrekidou A, Kosma I, Kotsanos N. Erosive Tooth Wear and Related Risk Factors in 8- and 14-Year-Old Greek Children. Caries Res. 2016; 50: 349-62.

27. Fleur P. The evolution of tooth wear indices. Clin Oral Invest. 2008; 12(1):15-9.

28. Singhal AC, Chandak S, Chamele J, Jain A, Gupta P, Thakur P. Indices for measuring dental erosion. Chhattisgarh J Health Sci. 2013;1:52-6.

29. Ganss C, Lussi A. Current erosion indexes-flawed or valid?. Clin Oral Invest. 2008; 12: 1-3.

30. López F, Castellanos L, Martín J, Llamas J, Segura J. Clinical measurement of tooth wear: Tooth Wear Indices. J Clin Exp Dent. 2012; 4: 48-53.

31. Caltrava L. Índices epidemiológicos del desgaste dental erosivo. RODYB. 2015; 4: 32-8.

32. Bartlett D, Ganss C, Lussi A. Basic Erosive Wear Examination (BEWE): a new scoring system for scientific and clinical needs. Clin Oral Invest. 2008; 12: 65-8.

33. Cavadini C, Siega-Riz AM, Popkin B. US adolescent food intake trends from 1965 to 1996. Arch Dis Child 2000; 83:18-24.

34. Bartlett D, Dugmore B. Pathological or physiological erosion - is there a relationship to age? Clin Oral Invest. 2008; 12: S27-S21.

35. Steinberg L. Risk taking in adolescence: what changes, and why? Ann N Y Acad Sci. 1021: 51-58, 2004.

36. Johanson AK, Omar R, Carlson G, Johanson A. Dental erosion and its growing importance in clinical practice: from past to present. Int J Dent. 2012; 2012:632907.

Recibido: $31 / 01 / 18$

Aceptado: 17/05/18

Correspondencia: Leslie Caroll Casas-Apayco.email: leslie.casas@upc.pe 